\title{
Data-driven Nonparametric Model Adaptive Precision Control for Linear Servo Systems
}

\author{
Rong-Min $\mathrm{Cao}^{1} \quad$ Zhong-Sheng Hou ${ }^{2} \quad$ Hui-Xing Zhou ${ }^{3}$ \\ ${ }^{1}$ School of Automation, Beijing Information Science \& Technology University, Beijing 100192, China \\ ${ }^{2}$ Advanced Control Systems Laboratory, Beijing Jiaotong University, Beijing 100044, China \\ ${ }^{3}$ College of Engineering, China Agricultural University, Beijing 100083, China
}

\begin{abstract}
Nowadays, high-precision motion controls are needed in modern manufacturing industry. A data-driven nonparametric model adaptive control (NMAC) method is proposed in this paper to control the position of a linear servo system. The controller design requires no information about the structure of linear servo system, and it is based on the estimation and forecasting of the pseudo-partial derivatives (PPD) which are estimated according to the voltage input and position output of the linear motor. The characteristics and operational mechanism of the permanent magnet synchronous linear motor (PMSLM) are introduced, and the proposed nonparametric model control strategy has been compared with the classic proportional-integral-derivative (PID) control algorithm. Several real-time experiments on the motion control system incorporating a permanent magnet synchronous linear motor showed that the nonparametric model adaptive control method improved the system's response to disturbances and its position-tracking precision, even for a nonlinear system with incompletely known dynamic characteristics.
\end{abstract}

Keywords: Data-driven control, nonparametric model adaptive control, precision motion control, permanent magnet synchronous linear motor, robustness.

\section{Introduction}

The scope of control theory and its application are expanding along with the rapid development of economy, science and technology. Due to the practical needs, lots of new problems are coming out, and new ideas, new methods for those problems are required. Moreover, as the interdisciplinary integration trend is further strengthened, more and more the applications using the control theory in different fields are increasing significantly. The computer numerical control (CNC) machine integrates mechanical system, electrical system, control system, information, materials, and the internet and it increasingly requires higher precision performance, faster dynamic response, less noise and less wear and more robustness.

The linear motor and its servo driven control technology in CNC machine improve the transmission structure of the machine and thus they are widely used in industrial applications ${ }^{[1-4]}$. However, the performance of linear motor may be affected by some factors while it is running, such as: the loads during the operation, and the changes of loads on the motor. The motor's parameters would also change during the operation. The friction in its linear guide ${ }^{[5,6]}$ is also required to be considered. All these disturbances and uncertainties would affect the accurate and precise control for linear motor and linear servo system. Furthermore, the conventional proportional-integral-derivative (PID) algorithm can be applied on the nonlinear system, the time-varying system, the coupling system and the system with uncertainties, but its control performances are not ideal, and its pa-

Manuscript received January 7, 2013; accepted November 27, 2013 This work was supported by Beijing Natural Science Foundation (No. 4142017), International Cooperation Project of National Natural Science Foundation of China (No.61120106009) and Beijing Science and Technology Commission Precision Machinery Projects (No. Z121100001612007). rameters are difficult to be tuned as well ${ }^{[7]}$. To overcome the above problems as well as to achieve the high performance on the linear motor or linear servo system ${ }^{[1-4]}$, the control strategy should be able to compensate the disturbances and reject the uncertainties. In recent years, a number of researchers have done researches on the advance control methods for the linear servo system which help to achieve high precision $^{[8-13]}$. In [9], an observer of disturbances based on the mathematical model was designed, which is able to compensate the disturbances in a certain bandwidth. However, the precise model which is not easy to obtain in the real application is required in this method. Moreover, there are several researches which have reported that the adaptive robust control methods are able to eliminate the effect of the external changes and system parameter changes effectively, as well as to increase the motor's dynamic stiffness, which help to suppress the aforementioned disturbances. However, it is not easy to carry out the nonlinear compensation of the permanent magnet synchronous linear motor (PMSLM). Additionally, these algorithms are complicated and always with a slow convergence of the control parameters. Therefore, their applications on the linear servo systems are limited. To solve these problems, the controller should be designed to have the features that can achieve good tracking performance, rapid dynamic response, strong anti-disturbance capability, and robustness. The traditional methods based on the model need to know controlled system order and model prior knowledge, they fail to deal with the control problems of linear servo system with structure time-varying, parameters time-varying, order time-varying and strong un-modeled dynamics, so these methods have been challenged.

Data-driven control method is based on the controller design that does not need to contain the information of the controlled system's mathematical model, it only uses the 
online and offline input/output (I/O) data of the controlled system which can be obtained through data processing. Under certain assumptions, data-driven control method has stability, convergence security and robustness ${ }^{[14]}$. The nonparametric model adaptive control (NMAC) method is a typical data-driven control method ${ }^{[15]}$.

In this paper, the data-driven, nonparametric model adaptive control of a linear servo system is proposed. The theoretical analysis and several experimental results presented in the following sections of this paper will show that this method is an effective method to remove the system disturbances and it is robust with regard to dynamic parameter changes. Moreover, the results also indicate that the application of this method could result in precise position control of a linear servo system.

\section{PMSLM: working principles}

\subsection{Working principles of the linear servo system}

This operation platform of the linear motor system is sourced from Zhengzhou Weiner Technology Co. Ltd. China. It consists of a U-shaped PMSLM, mechanical motion platform, precision linear guide rail, encoder sensing device, digital servo driver and computer. The structure diagram of the linear servo system is shown in Fig. 1. The operating parameters of this PMSLM are as follows: maximum speed is $1 \mathrm{~ms}^{-1}$; maximum acceleration is $60 \mathrm{~ms}^{-2}$ : maximum stroke is $380 \mathrm{~mm}$; nominal thrust is $30 \mathrm{~N}$; and peak thrust is $80 \mathrm{~N}$.

The nonparametric model adaptive linear servo system used for this research is a double closed loop system, consisting of a speed control loop and followed by a position control loop. The speed control loop is established inside the linear motor's driver while the position control loop is established by the NMAC. The system's operating princi- ples are shown in Fig. 2. This system achieves the capability of motion control system through the use of a computer with a control card of the hardware-in-loop (HIL) (sourced from cSPACE), a linear servo driver, an encoder and a linear motor.

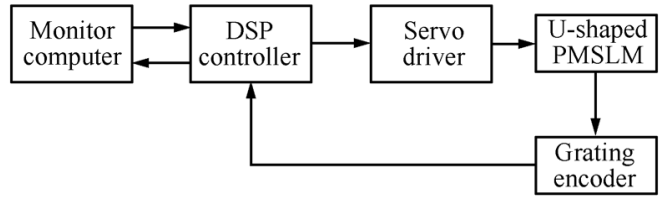

Fig. 1 Structure diagram of the linear servo system

The USB interface in Fig. 2 connects the computer with the cSPACE control card. Its function is to transfer the NMAC algorithm DSP code onto the DSP of the cSPACE control card.

The RS232 interface in Fig. 2 connects the computer with the cSPACE control card. Its function is to monitor the motor output signals from the cSPACE to the computer and to control the motor's start and stop operations.

The encoder interface in Fig. 2 connects the encoder with the cSPACE control card. Its function is to send the position information of the linear motor to the DSP of the cSPACE control card.

Working procedure 1.

The designed NMAC algorithm is established by Matlab/Simulink, the I/O interfaces are replaced by cSPACE's Simulink hardware module. The compiler module compiles the NMAC algorithm into the DSP code which is then downloaded to the DSP of the cSPACE control card via the USB interface. Each hardware interface module is corresponded to the hardware interface of the cSPACE control card. Table 1 is the description of the hardware interface modules functions. Table 1 illustrates the function of each interface module in Fig. 2; these modules are a "bridge"

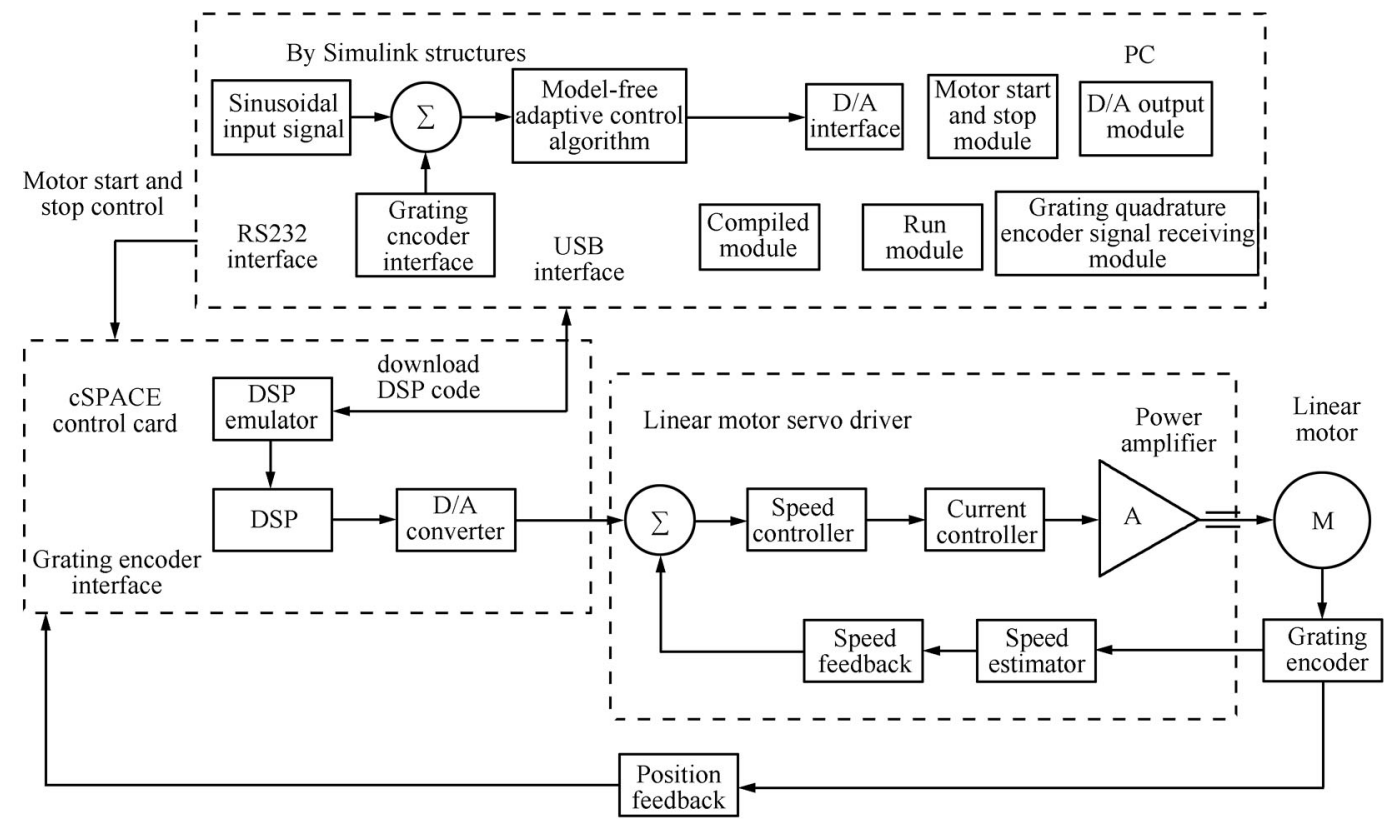

Fig. 2 Operation principle for linear servo system 
Table 1 Function descriptions for hardware interface modules

\begin{tabular}{ll}
\hline \multicolumn{1}{c}{ Type of interface module } & Function for interface module \\
\hline $\begin{array}{l}\text { Grating quadrature encoder signal } \\
\text { receiving module }\end{array}$ & To detect the position signal of linear motor and output pulse number \\
D/A output module & 16-bit analog to digital conversion, output range is $(-10 \mathrm{~V},+10 \mathrm{~V})$ \\
Motor start and stop module & PC control the motor start and stop by the serial port \\
Compiling module & The control algorithm files based on Simulink program are compiled into executable code \\
Run module & Automatically run the generated target code downloaded to the cSPACE control card \\
& based on Matlab/Simulink, and automatically start the cSPACE monitor screen \\
\hline
\end{tabular}

between the software and the hardware, which links the Simulink to the hardware system. The descriptions mentioned above are the functions of the cSPACE.

\section{Working procedure 2.}

The position signals relating to the linear motor are collected through the encoder and sent to the DSP of the cSPACE control card. Then they are compared with the input signals of the desired sine position and thus obtain the error signals. The error signals are then processed by NMAC algorithm to obtain the control signal for the subsequent transmission to the servo system of the linear motor. The resolution of the detector is $5 \mu \mathrm{m}$.

\section{Working procedure 3 .}

The control signals from the DSP of the cSPACE control card are amplified by the servo driver which is used to drive the linear motor. The driver was sourced from the Elmo Company, Israel. To obtain the possible maximum output, the driver is set to work under the speed mode whereby the internal speed control loop - comprising the speed controller, the speed feedback, and speed estimation — is used to control the speed.

\subsection{Characteristic analysis of permanent magnet synchronous linear motor}

The kinetics of the PMSLM in the control system is usually divided into the constituent linear and the non-linear parts: The linear part can be represented by the kinetic electromechanical equations:

$$
\begin{aligned}
& \ddot{x}=-\frac{K_{1}}{M} \dot{x}+\frac{K_{2}}{M} u-\frac{1}{M} F_{\text {load }} \\
& K_{1}=\frac{K_{e} K_{f}+R_{a} D}{R_{a}}, \quad K_{2}=\frac{K_{f}}{R_{a}}
\end{aligned}
$$

where $x$ is the position of the linear motor, $M, D$ and $F_{\text {load }}$ are the mechanical parameters of the motor, representing inertial, viscous, and load forces, respectively, $u$ and $R_{a}$ are the motor's DC voltage and armature resistance, respectively, $K_{f}$ is the energy transformation constant, and $K_{e}$ is the back electromotive force (EMF) constant.

Using (1) and (2), the linear part of the PMSLM can be considered as a 2nd order dynamic model, representing the linear relationship among the motor parameters. The dominant linear model does not include the extraneous nonlinear effects which may be presented in the real system. Among them, there are two prominent non-linear effects associated with PMSLM. They are due to the ripple and the friction forces, arising from the magnetic structure of PMSLM and other physical imperfections ${ }^{[5]}$. A first-order model for the force ripple can be described as a periodic sinusoidal type signal:

$$
f_{\text {ripple }}=b_{1} \sin \left(w_{0} x\right)
$$

where $b_{1}$ is the amplitude of the force ripple and $w_{0}$ is the angular velocity of force ripple.

In the actual operation, because of the change of magnetic field, the force ripple is more complex, it has different higher order dynamics model, but there are same period and amplitude, so easy comparison.

The frictional force includes Coulomb friction, viscous resistance, and static friction components ${ }^{[6]}$ :

$$
f_{\text {friction }}=\left(f_{c}+\left(f_{s}-f_{c}\right) \mathrm{e}^{-\left(\frac{\dot{x}}{\dot{x}_{s}}\right)^{\delta}}+f_{v} \dot{x}\right) \operatorname{sgn}(\dot{x})
$$

where $f_{s}$ is the static friction force; $f_{c}$ is the maximum value of the Coulomb friction; $\dot{x}_{s}$ is the lubrication parameter; $f_{v}$ is the load parameter; and $\delta$ is an additional empirical parameter.

Equations (3) and (4) show that the magnetic thrust fluctuation and the friction are mainly determined by the speed and the position of the motor. Both of them have a nonlinear relationship with the speed and position. During the motion, due to the changes in speed and position, the thrust fluctuation and the friction will change, thus increasing the difficulties in compensating the thrust fluctuations. Through this analysis, it can be seen that the PMSLM is a nonlinear system with complex structure, variable parameters, as well as variable structures, order numbers, and parameters.

\subsection{Linear motor control system software framework}

The servo system of permanent magnet synchronous linear motor is not only required to have high performance, but it also needs that the system software framework is functioning perfectly to ensure a convenient debugging servo system. To meet different requirements of the servo control system, the hardware part of the experiment system is equipped with a special software development platform, so that it can ensure that the operation of the whole servo system is effective. The software platform of the system framework is shown in Fig. 3.

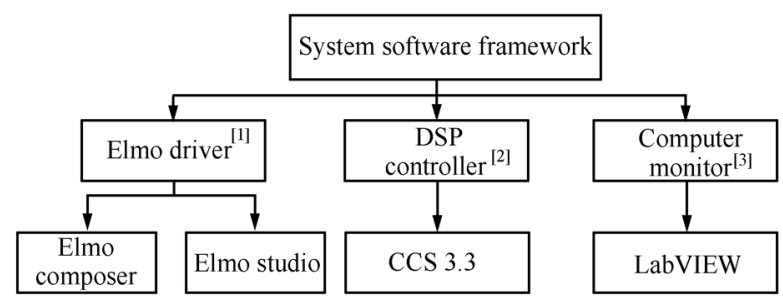

Fig. 3 System software platform framework

The Elmo composer is used to realize serial communication between driver and computer, to complete the driving 
mode selection, and to implement the manual debugging on the target machine.

The Elmo drive is used to drive servo motor, which has a simple programming function so that it can achieve some simple control of servo motor.

The Elmo studio is to manage the driver programming, compiling and communication.

The code composer studio (CCS) from TI Company is a complete DSP integrated development environment. In this system, the CCS3.3 is used for the programming of DSP (TMS320F2812).

In this paper, a monitoring interface for the servo motor control system is implemented in LabVIEW.

\section{Nonparametric model adaptive con- trol for a linear servo system}

\subsection{Basic theory of the nonparametric models adaptive control system}

The essence of NMAC is to replace the general discrete time nonlinear system with a compact format linearization real time model at the current working position in the controlled system. It uses the I/O data from the controlled system to estimate the psoudo-partial-derivative (PPD) in a compact format linearization, dynamic, linear and online model. And finally a nonparametric model adaptive controller for such a nonlinear system is accomplished ${ }^{[15]}$. The general discrete time single input single output (SISO) nonlinear system can be effectively represented by

$$
\begin{aligned}
y(k+1)= & f\left(y(k), y(k-1), \cdots, y\left(k-n_{y}\right), u(k),\right. \\
& \left.u(k-1), \cdots, u\left(k-n_{u}\right)\right)
\end{aligned}
$$

where $u(k)$ and $y(k)$ are the system's input and output at the moment $k ; n_{y}$ and $n_{u}$ are unknown orders of the system; and $f(\cdot)$ is an unknown nonlinear function.

The NMAC is based on the following assumptions made from the system:

Assumption 1. The system (5) is observable and controllable in following meanings, that is, for the expected bounded system output signal $y^{*}(k+1)$, there exists a bounded feasible control input signal which drives the system output equal to the expected output.

Assumption 2. The partial derivative of $f(\cdots)$ with respect to control input $u(k)$ is continuous.

Assumption 3. The system (5) is generalized Lipschitz, which is satisfying $|\Delta y(k+1)| \leqslant b|\Delta u(k)|$ for $k$ and $\Delta u(k) \neq 0$, where $\Delta y(k+1)=y(k+1)-y(k), \Delta u(k)=$ $u(k)-u(k-1), b$ is a constant.

Assumption 3 is a limit on the rate of change of the system output. Obviously, it includes a class of nonlinear plant.

Theorem 1. The nonlinear system (5) satisfies the Assumptions $1-3$ thus there must exist $\phi(k)$, called PPD, when $\Delta u(k) \neq 0$

$$
\Delta y(k+1)=\phi(k) \Delta u(k)
$$

where $\varphi(k)$ is the system's PPD, $|\phi(k)| \leqslant b$ is bounded, and $b$ is a constant.
Verification of this linearization is based on [15]. Compared with other linearization methods, the compact format linearization method has features as follows:

1) No requirements for either a mathematical model of the controlled system and its orders, or the associated time delay.

2) No reference trajectory is needed for the controlled system.

3) No empirical knowledge of the controlled system is needed.

4) The linearization model is data-based with a view of a control system design with simple structures and few parameters. It is a real-time incremental model.

In the design of the NMAC algorithm, the adjustable parameter $\lambda$ should be added to the control algorithm so as to limit the change of $\Delta u(k)$ as well as guarantee that $\Delta u(k) \neq 0$. The above method is operated by the minimized weighting error-forecasting criterion function: The nonparametric models adaptive control algorithm based on the compact format linearization is obtained below:

$$
u(k)=u(k-1)+\frac{\rho_{k} \phi(k)}{\lambda+\phi(k)^{2}}\left[y^{*}(k+1)-y(k)\right]
$$

where $\rho_{k}$ is the step series, $\rho_{k} \in(0,2)$, and $\lambda$ is the penalty factor of the control input change. Besides the linear compensation for the nonlinear systems, the change of in PPD may also need to be constrained. To avoid rapid changes in the estimation or undue sensitivity to some mutating samples among the parameters, the estimation criterion function is proposed as follows:

$$
\begin{aligned}
J(\phi(k))= & {\left[\left(y^{0}(k)-y(k-1)-\phi(k) \Delta u(k-1)\right)^{2}+\right.} \\
& \left.\mu(\phi(k)-\hat{\phi}(k-1))^{2}\right]
\end{aligned}
$$

where $y^{0}(k)$ is the actual output; $\mu$ is a penalty factor of the parameter's estimation variable, they can constrain the linear replacement range of the nonlinear system. Due to the consideration of the $k$-th sampling moment in (8), the parameter estimation algorithm derived from the criterion function has the ability to track real time variations in the parameter(s). By the minimization derivation ${ }^{[15]}$, the PPD estimation algorithm is obtained by:

$$
\begin{aligned}
\hat{\phi}(k)= & \hat{\phi}(k-1)+\frac{\eta_{k} \Delta u(k-1)}{\mu+|\Delta u(k-1)|^{2}} \times \\
& (\Delta y(k)-\hat{\phi}(k-1) \Delta u(k-1)) \\
\hat{\varphi}(k)= & \hat{\varphi}(1), \text { if } \hat{\phi}(k) \leqslant \varepsilon \text { or }|\Delta u(k-1)| \leqslant \varepsilon
\end{aligned}
$$

where $\eta_{k}$ is the step series, $\eta_{k} \in(0,2), \varepsilon$ is the minimum positive number, and $\hat{\phi}(1)$ is the initial value of $\hat{\phi}(k)$. As can be seen in (7), the control law has no relation with the mathematical model structure and the system orders. Only the system I/O data is required for forming the basis for the subsequent design. This method may realize the parameter adaptive control and structure adaptive control. Due to the unique, single online adjustment parameter (PPD), the calculation is straightforward. 


\subsection{NMA controller design of the linear servo system}

NMAC has been widely used in refineries, chemical industry, electrical and electronics industry, traffic and transportation ${ }^{[16]}$, DC motor and chemical-industrial moulding applications ${ }^{[12]}$. Based on the analysis of the undefined nonlinear feature of PMSLM, the NMAC method with the compact format linearization is proposed in this research since it is suitable for position controller design of the linear motor servo system. In this way, the disturbances are able to be suppressed and the high precision motion control can be thus realized.

Equation (5) shows the behavior of a typical servo system for the PMSLM, $u(k)$ and $y(k)$ are the input voltage and output position, respectively at the $k$-th moment. $n_{y}$ and $n_{u}$ are the uncertain order numbers of the servo system, $f(\cdot)$ is the uncertain nonlinear function of the servo system.

The linear servo system fulfills the following three conditions:

1) The input voltage and the output position are observable and controlled. For the output signal which is a desired sine position with certain amplitude, there is always a corresponding voltage input signal that makes the position output to follow the desired sine input.

2) The PPD of the voltage control input $u(k)$ for the unknown non-linear function in the linear servo system is continuous. When the input control voltage is within the allowable range of the motor, the voltage increment would cause corresponding changes in the position output increment.

3) Changes in the bounded input voltage cause changes in the bounded output position; the input voltage in the allowed range of the motor can bring in changes of the input position within the allowable range for the motor. This acts as a constraint on the position output of the linear servo system.

According to the compact format dynamic linearization model in (6), the PPD can be regarded as a slow real time parameter when both the sampling period and the voltage variable $\Delta u(k)$ are negligibly small, therefore, the relationship between PPD and $u(k)$ can be omitted. The sampling period is $0.005 \mathrm{~s}$, and the voltage variable of the motor $\Delta u(k)$ is therefore deemed to be sufficiently small. Thus, the design of the adaptive controller for the linear servo system is feasible.

The control algorithm (7) shows that the response speed of the servo system is faster when the weighting factor $\lambda$ is smaller. Meanwhile, the overshoot may occur and the motor may run out-of-balance. In contrary, the response speed is slower when $\lambda$ is larger. The influence of $\lambda$ on the system dynamics will be validated in the experiments.

According to the estimation algorithm, the early restart algorithm (10) aims to improve the tracking ability of the algorithm of (9) for real time parameters. Equation (10) can prevent the voltage variable $\Delta u(k)$ from becoming zero (at which point the motor system would cease to function). Parameters for the design of the linear servo system are as follows: the step series $\eta_{k}$ is $1.5, \rho_{k}$ is 0.01 , penalty factor $\mu$ is $1, \varepsilon$ is $0.0014 ; \hat{\phi}(1)$ is the initial value of $\hat{\phi}(k)$, the initial values are 2 and 0.5 , respectively, when the system is reset.

\subsection{Implementation of cSPACE Simulink for the NMAC algorithm}

Fig. 4 shows the algorithm framework established by cSPACE/Simulink based on the aforementioned NMAC and PPD algorithms (the PID algorithm framework is not given). The hardware interface module was connected to the cSPACE control card, and NMA position control was achieved by the working principles in Fig. 2. Modules from "WM-Read 1" to "WM-Read 4" provide the observation values, which can revise the parameters of the NMAC algorithm and the amplitude of the sine signal of the desired position input. And the real-time display graphics of the control results are provided for the observation of running effect of the linear servo system. Table 2 introduces interface module functions of the NMAC algorithm. In Fig. 4,

Table 2 Introduction of module functions of the NMAC algorithm

\begin{tabular}{ll}
\hline \multicolumn{1}{c}{ Name of module } & \multicolumn{1}{c}{ Function } \\
\hline cSPACE WM-Write 1 module & Adjust amplitude of the sinusoidal signal on-line \\
cSPACE WM-Write 2 module & Adjust $\lambda$ in the control algorithm $(7)$ of the nonparametric model adaptive control online \\
cSPACE WM-Write 3 module & Adjust $\rho_{k}$ in the control algorithm (7) of the nonparametric models adaptive control online \\
cSPACE WM-Write 4 module & Calculate the initial value of PPD $\hat{\varphi}(k)$ in the online estimation algorithm (9) of NMAC \\
cSPACE WM-Read 1 module & To observe the desired position input value of the linear motor \\
cSPACE WM-Read 2 module & To observe the output position value of the linear motor \\
cSPACE WM-Read 3 module & To observe the output position error-value of the linear motor \\
cSPACE WM-Read 4 module & To observe the PPD value of NMAC \\
F2812 eZdsp & TMS320F2812DSP control card; needed by every Simulink control algorithm file \\
Run & Simulink file run button \\
Build & Simulink file compilation button \\
WM-Motor on/off & Motor start and stop button \\
WM-DAC 1 & Connect Simulink file with cSPACE interface card, to achieve D/A translation \\
WM-Encode 2 & Connect encoder with PC, to achieve position signal collection \\
Sine wave & Input sine signal \\
\hline
\end{tabular}




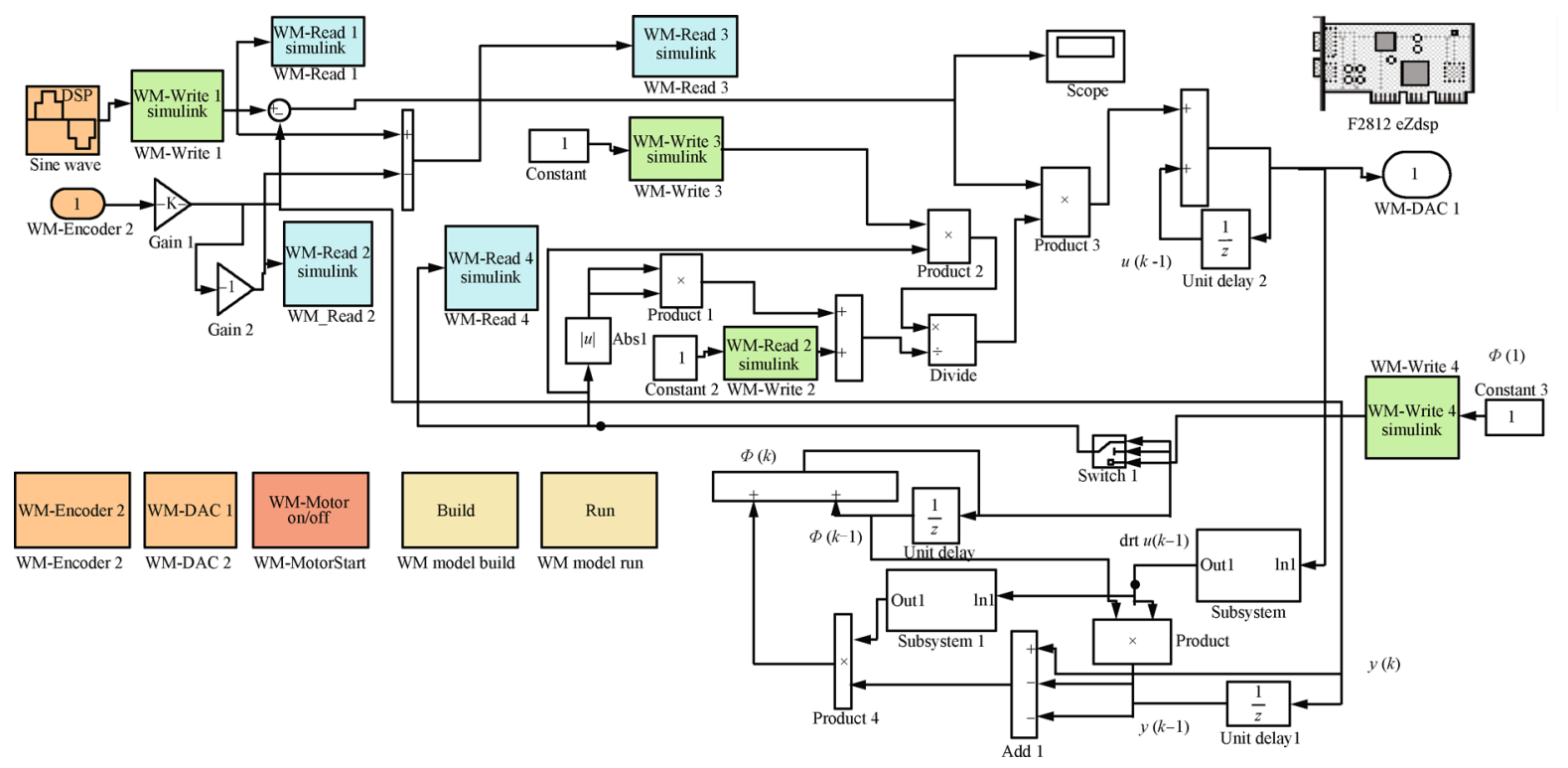

Fig. 4 NMAC method for linear servo system

a PPD restart algorithm (10) is achieved by a switch in Simulink in order to reflect the choice of $\varepsilon$. In this way, excessively low voltages are avoided, resulting in a stable system. Fig. 4 shows the hardware-in-loop (sourced from cSPACE), software (NMAC algorithm file based on Simulink) and the hardware (drive, motor, grating encoder and cSPACE control card) and their connections.

\section{Experiments}

The experimental work is divided into two parts: firstly, the driver feature tests, secondly, the servo system control test $^{[17]}$.

\subsection{Characterization testing and analysis of the linear servo driver}

The dynamic response of the linear servo system directly determines the performance of the NMAC position control. Three main indices are used to evaluate the linear servo system behavior: the maximum overshoot, the steady-state error, and the bandwidth. The aforementioned Elmo composer was used to set and adjust the parameters of the servo driver of the motor through RS232 interface before testing and analyzing the operational characteristics of the motor. Those frequency-domain properties measured in the experiment are shown in Fig. 5. In the closed-loop amplitudefrequency characteristic, when the frequency decreases below $3 \mathrm{~dB}$ from 0 , the corresponding frequency is called bandwidth frequency. The bandwidth definition indicates that if the sinusoidal input signal is higher than the bandwidth frequency, the system output will show significant attenuation. As can be seen from the phase-frequency feature in Fig. 5, the speed inner-loop system has larger bandwidth which is about $200 \mathrm{~Hz}$. During the experiments, the sine input signals ranging from $0.2 \mathrm{~Hz}$ to $1 \mathrm{~Hz}$ are used. Because the bandwidth is much larger than the input signal frequency, the response speed is faster and the control signal-tracking ability can be improved.

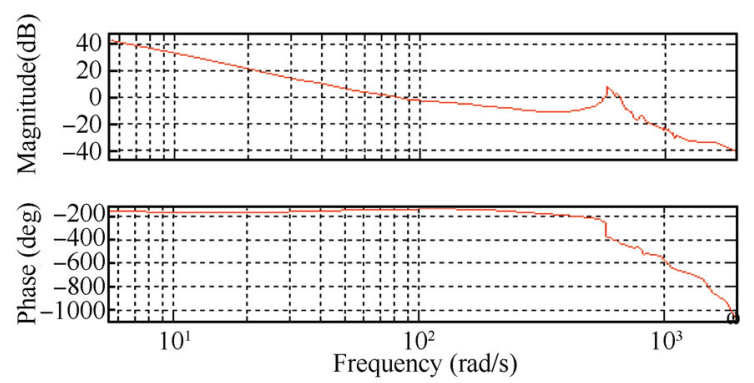

Fig. 5 frequency response characteristics for the linear servo driver

Fig. 6 shows the current input and the speed tracking performance of the linear motor when the speed inner-loop system is operating in response to the step input. Since the resolution of the encoder was 5 micrometers (one pulse is equivalent to $0.5 \mathrm{~m}$ ), the average speed is $0.1 \mathrm{~ms}^{-1}$ when the desired step speed input is 20000 pulses per second.
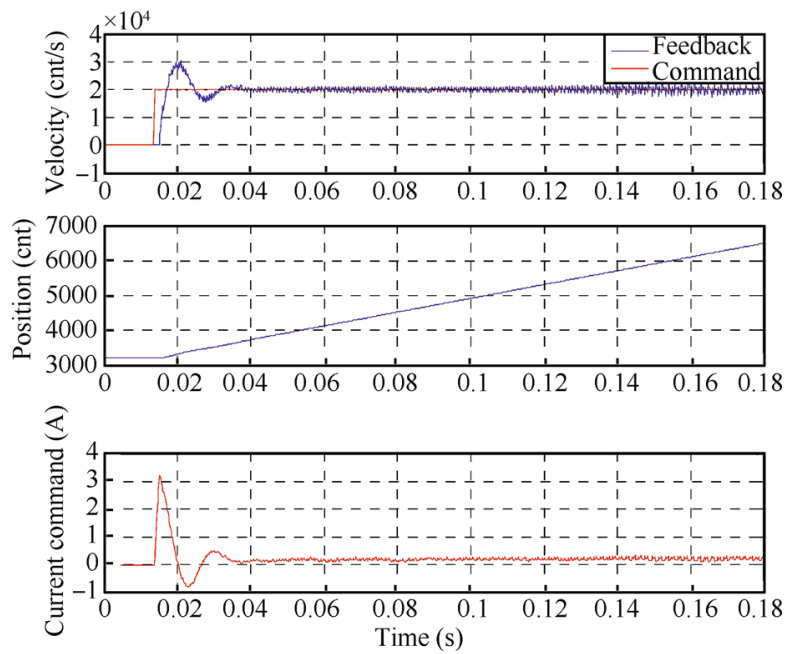

Fig. 6 Current input and speed tracking characteristics for the linear servo driver 
Although there are some overshoots in Fig.6, the response speed is fast, the settling time is short, and the steadystate error is zero. Thus, it can ensure the speed inner-loop stability of servo system. Based on the speed inner-loop stability, this paper mainly focuses on the research of the position precision control, same as servo system the position outer-loop control.

\subsection{Control experiment for the linear servo system}

The purpose of this experiment is to apply the NMAC method based on the tight-format linearization. For comparison, the PID algorithm was also applied to the position control of the linear servo system. The aim is to make the linear motor reciprocate in-line with different input signal frequencies (various speeds), different distances (various accelerations) and different disturbances (no-load and loaded), and then to analyze the system position-tracking error, to check the ability of the system to suppress disturbances, and to verify the robustness of the NMAC controller and its precision control feature.

According to the feature indices of the hardware, experiments involving PID and NMAC algorithms were both performed at different speeds (different frequencies of the sine signal of the desired position input) and different distances (different amplitudes, in the range $3 \mathrm{~mm}$ to $190 \mathrm{~mm}$, of the sine signal of the desired position input). The sampling time was $0.005 \mathrm{~s}$; the sine signal amplitude of the desired position input was $90 \mathrm{~mm}$ (with a maximum distance of $180 \mathrm{~mm}$ ). Three configurations are summarized in Table 3.

Case A. Parameters were adjusted to their optimal values by the PID algorithm $(0.9,18$, and 0 , respectively): the tracking error was within $0.6 \mathrm{~mm}$, as can be seen in Fig. 7 (a). Then the NMAC algorithm was employed instead of the PID algorithm, and the closed-loop response and stability can be obtained by adjusting the weighting constant $\lambda$. In this way, stable and precise tracking responses by using NMAC are obtained making it superior to PID control. The tracking error while using NMAC is within $0.2 \mathrm{~mm}$, as shown in Fig. 7 (b): At $\lambda=4$, the initial value of $\varphi(k)$ was $\varphi(1)=2$.

The amplitude of the sine signal was then reduced to $30 \mathrm{~mm}$. For the short-distance movements of the motor, the position errors of PID and NMAC showed few differences which is within $\pm 0.01 \mathrm{~mm}$. When the amplitude of the sine signal was increased to $150 \mathrm{~mm}$, the distance moved by the motor became longer, i.e., $300 \mathrm{~mm}$, and the control effect is more similar.

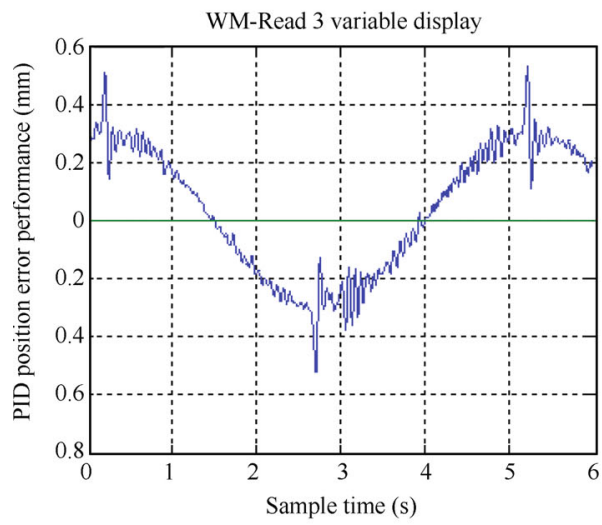

(a) PID position error performance

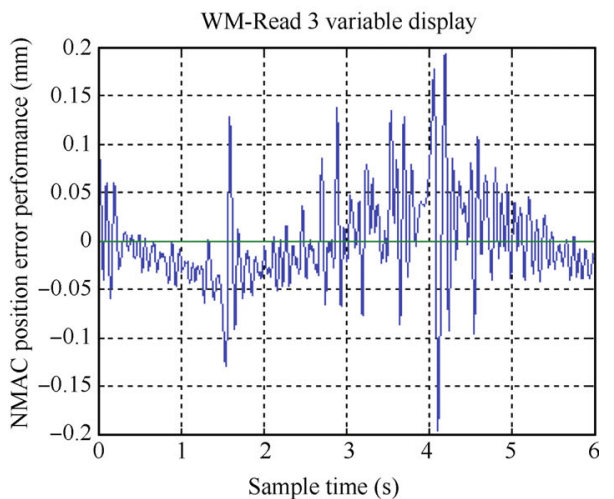

(b) NMAC position error performance

Fig. 7 Case A: feature comparison of PID and NMAC

In the case of slow speed and no-load, both the PID and NMAC guaranteed a stable closed-loop system, because position trajectory of linear motor is a sinusoidally, two algorithms can ensure the output position change tracking input position change accurately. Even if the locus and characteristics of the motor's movements were changed (with respect to acceleration, current, thrust fluctuation, etc.), these two algorithms still achieve acceptable control performances. On the other hand, the PID algorithm had excessive adjusting parameters, and any changes thereto may affect the quality of control. The NMAC algorithm is only required to tune one parameter $\lambda$ which is easy to be tuned.

Table 3 Experimental division of PID and NMAC

\begin{tabular}{|c|c|c|c|}
\hline Configuration & Input signal of the desired position & Load disturbance & Purpose of the experiment \\
\hline $\mathrm{A}$ & $\begin{array}{l}0.2 \mathrm{~Hz} \text { sine wave (average speed, } \\
\text { about } 72 \mathrm{~mm} \cdot \mathrm{s}^{-1}\end{array}$ & No-load & $\begin{array}{l}\text { To verify the position-tracking effect of the } \\
\text { two algorithms at low operating speed }\end{array}$ \\
\hline $\mathrm{B}$ & $\begin{array}{l}1 \mathrm{~Hz} \text { sine wave (average speed, } \\
\text { about } 360 \mathrm{~mm} \cdot \mathrm{s}^{-1} \text { ) }\end{array}$ & No-load & $\begin{array}{l}\text { To verify the adaptability of the two methods to the } \\
\text { position tracking and their control precision when } \\
\text { situation } \mathrm{A} \text { is stable at low operating speed }\end{array}$ \\
\hline $\mathrm{C}$ & $\begin{array}{l}1 \mathrm{~Hz} \text { sine wave (average speed, } \\
\text { about } 360 \mathrm{~mm} \cdot \mathrm{s}^{-1} \text { ) }\end{array}$ & $\begin{array}{l}2 \mathrm{~kg} \text { added when the } \\
\text { methods when the load } \\
\text { operation was stable }\end{array}$ & $\begin{array}{l}\text { To verify the robustness of the two } \\
\text { disturbance changed }\end{array}$ \\
\hline
\end{tabular}


The maximum position error and the maximum variability of the position error occurred at the moment while the motor switched its direction. The resolution of the encoder was $5 \mu \mathrm{m}$, but the position error control results did not achieve micrometer's level. The main reasons are as follows:

Note 1. Although the real-time simulation control on the hardware-in-loop is a common method for the design of the control system, but it has some limitations. The encoding efficiency, data exchange pattern, and the simulator performance affect the data transmission bandwidth, which may limit the improvement of the sampling frequency.

Note 2. The motor time constant of the servo system refers to the length of the transition time of the motor, it reflects the changing speed of the system, which can be within $1 \mathrm{~ms}$. The sampling time in this research was $5 \mathrm{~ms}$. Due to the low sampling frequency, the dynamic characteristic of the servo system is affected.

Therefore, as long as the NMAC scheme is applied in the DSP and running at a full speed instead of hardware in the loop simulation, it can make the position error control in micrometers.

Case B. The sine signal frequency of the desired position was increased from $0.2 \mathrm{~Hz}$ to $1 \mathrm{~Hz}$, which means that the operating speed of the linear motor is increased by five times faster than the Case A: Fig. 8 (a) shows the position error of the PID control system at this moment relative to the optimal parameters $(1,24,0)$. The maximum error is $3 \mathrm{~mm}$, which is a big tracking error with poor control precision. However, the control performance of the NMAC algorithm was good. The error is within $0.4 \mathrm{~mm}$, as can be seen in Fig. 8 (b). At this moment, $\lambda$ was adjusted to 1.3 and the initial value of PPD became $\hat{\phi}(1)=2$. The analysis of PMSLM showed that, the current drawn by the driving motor changed when the speed changed. Thus the real time disturbance caused by the current change significantly affected the motor's dynamics. Besides, the quasi-perfect adaptability to the changing model parameters verified that the control principle of NMAC is independent of the model, and its PPD is a real-time parameter. Due to its continuous changes, the adaptive control method could adjust the relations between the position and the voltage, as can be seen in Fig. $8(\mathrm{c})$. The PID and NMAC tracking performances are shown in Figs. 7 (c) and $8(\mathrm{~d})$. In the case of load disturbance, the advantage of NMAC control effect is better obviously.

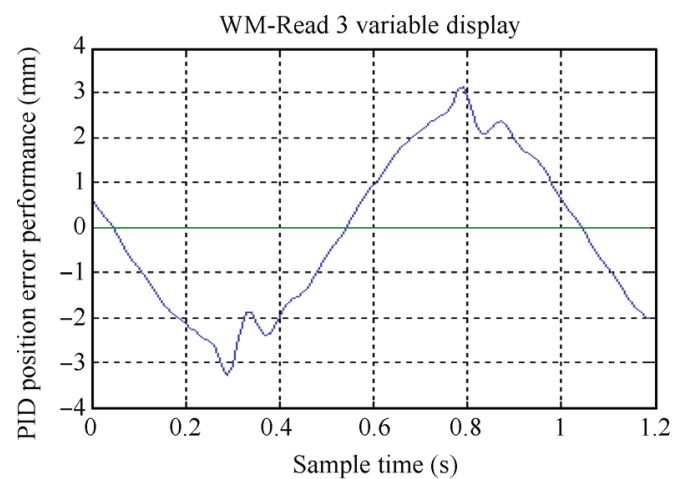

(a) PID position error performance

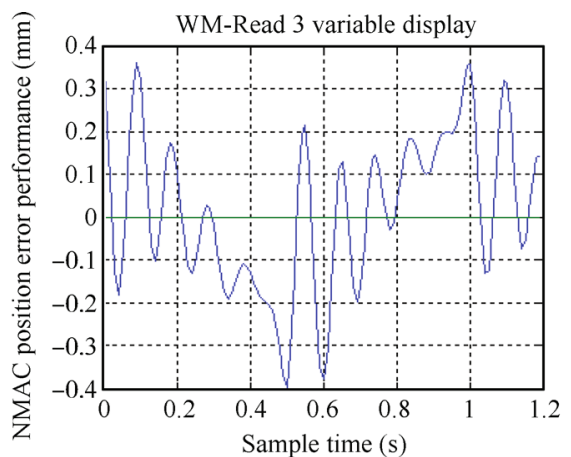

(b) NMAC position error performance

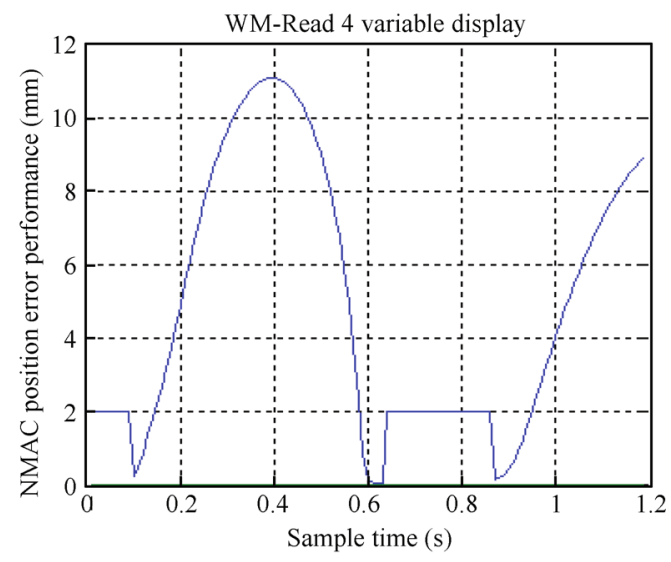

(c) NMAC pseudo-partial derivatives performance

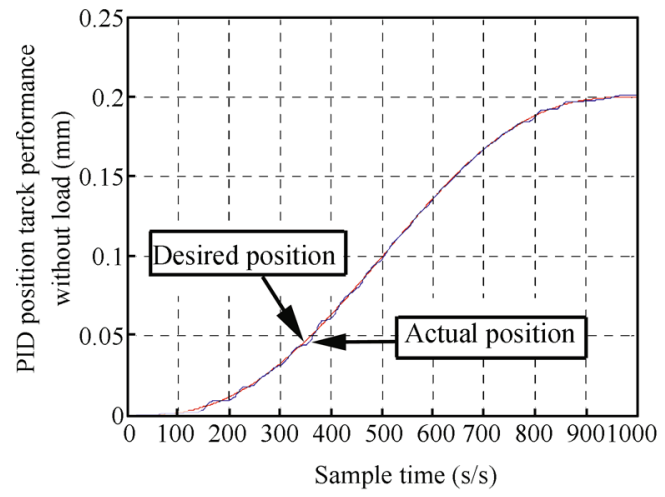

(d) PID track performance

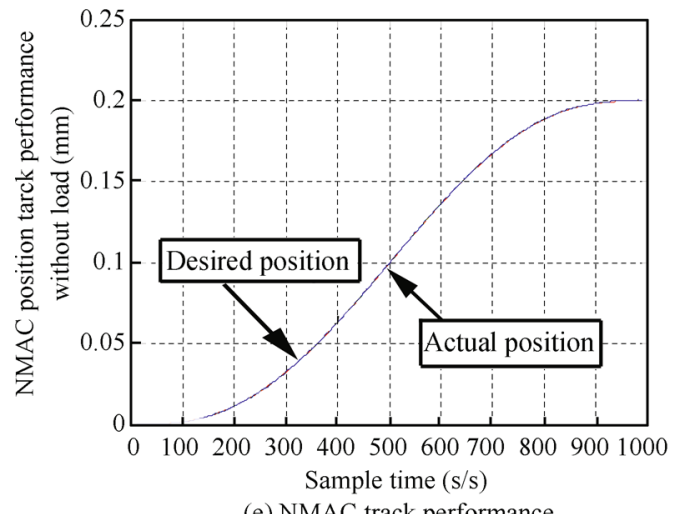

(e) NMAC track performance

Fig. 8 Case B: Feature comparison of PID and NMAC algorithms

Other parameters were kept constant with $\lambda>8$, 
then the position output brought about significant phasedifferences as high as 180 degrees. When $\lambda>10$, the system became unstable. This is because the increasing $\lambda$ value causes the voltage to tend to zero, and thus the PPD is no longer a real-time parameter. Therefore, it lost the ability of adjusting the parameters for adapting to the changes in either voltage or position. When $\lambda$ is decreased, the output changed to be a triangular wave and even clipping of the waveform to form a flat-topped profile occurred with significant position-tracking errors, as seen in Fig. 9.

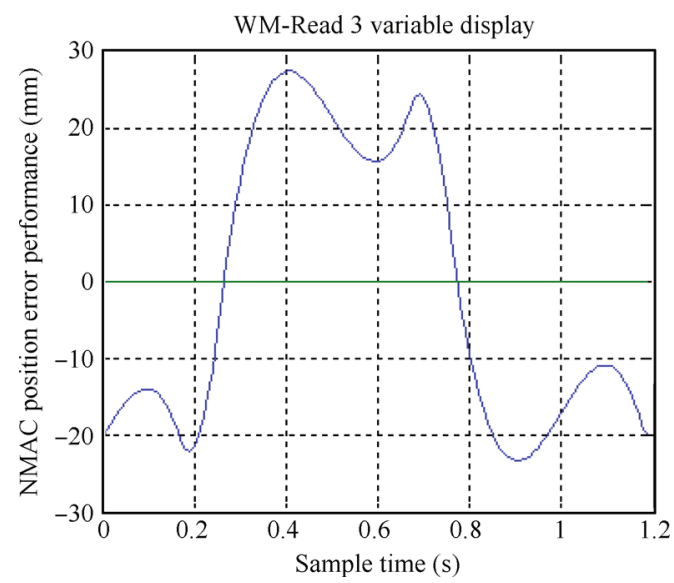

Fig. 9 Influence of $\lambda$ changes on the system's dynamic properties in NMAC

For $\lambda<0.5$, the system began to vibrate because $\lambda$ was excessively small, thereby causing a significant voltage variability/instability. Therefore, the changes in $\lambda$ affected the system's dynamics, as predicted by the aforementioned theories. As can be seen from the perspective of these changes in $\lambda, \lambda$ is insensitive to the other parameters and its potential range of adjustment is wide. It implies that the system is robust.

Case C. The parameters which are applicable to Case $\mathrm{B}$ were kept the same. However, the servo system was subjected to an additional weight of $2 \mathrm{~kg}$. The initial value $\hat{\phi}(1)$ of the PPD was reset to be 0.5 , and then the error is within $1 \mathrm{~mm}$, as can be seen in Fig. 10 .

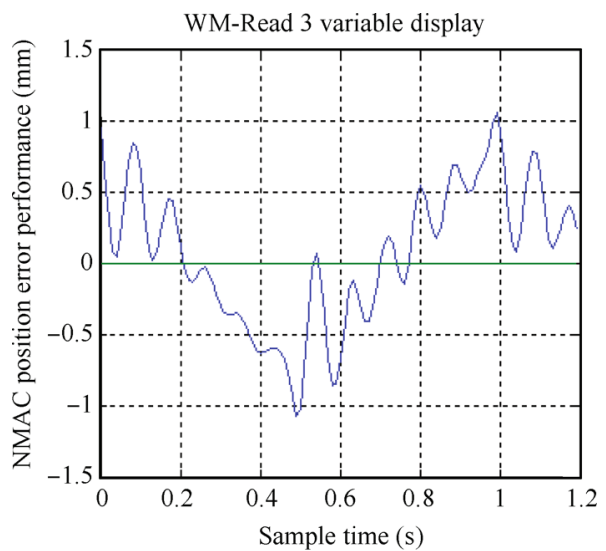

(a) NMAC position error performance

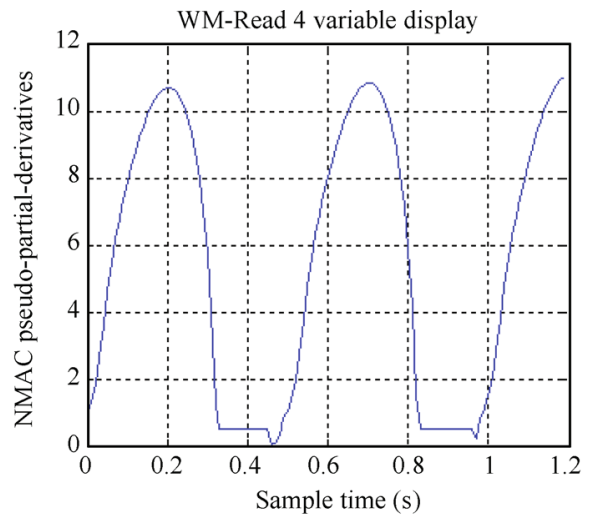

(b) NMAC pseudo-partial-derivatives performance

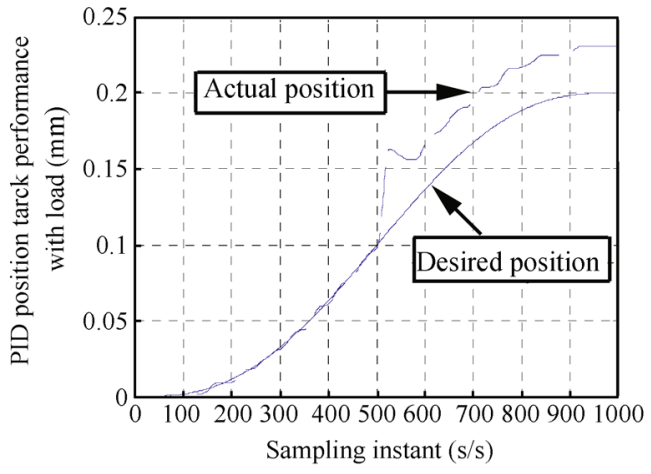

(c) PID track performance

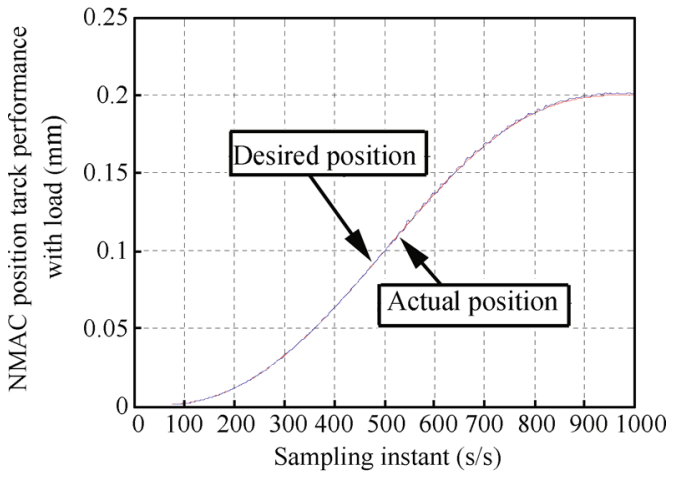

(d) NMAC track performance

Fig. 10 Control effect of the loaded NMAC

After the loading, the system could not be controlled by the PID algorithm no matter how its parameters are adjusted. The linear motor vibrated excessively, until its mover reached the end of its travel range, and the motor stopped working. As can be seen from the perspective of the electromechanical formulation given in (1) for the PMSLM, the load was related to the voltage, position, speed, acceleration, etc.. The load would cause corresponding changes of these parameters. Under such changes, NMAC still retained its performance, showing its independence from the model as well as affording good control of the non-linear, realtime system. The PID and NMAC tracking performances are shown in Figs. 10 (c) and (d). It can be observed that NMAC position tracking performance is much better than PID .

Under the identical conditions, the position tracking er- 
ror of NMAC is $0.4 \mathrm{~mm}-2.6 \mathrm{~mm}$ which is smaller than the one obtained by PID method in real time experiments.

\section{Conclusions}

The nonparametric model adaptive compact-format linearization control method based on the data-driven was applied to a linear servo system. Since the controller is designed with less dependency on the model parameters, the pseudo-partial derivatives could automatically regulate both the motor drive voltage and the position output, when the motor parameters (which included: speed, acceleration, external load perturbation, etc.) varied. This showed a good robustness of the proposed control system. Moreover, the NMAC algorithm showed a good adaptability and robustness when it was applied to different signal frequencies (velocity) or linear motor stroke (acceleration) variations.

\section{References}

[1] T. H. Lee, K. K. Tan, S. N. Huang. Adaptive friction compensation with a dynamical friction model. IEEE/ASME Transactions on Mechatronics, vol.16, no. 1, pp.133-138, 2011.

[2] M. Allouche, M. Chaabane, M. Souissi, D. Mehdi, F. Tadeo. State feedback tracking control for indirect field-oriented induction motor using fuzzy approach. International Journal of Automation and Computing, vol.10, no. 2, pp.99-110, 2013.

[3] K. Jamoussi, L. Chrifi-Alaoui, H. Benderradji, A. El Hajjaji, M. Ouali. Robust sliding mode control using adaptive switching gain for induction motors. International Journal of Automation and Computing, vol. 10, no. 4, pp. 303-311, 2013.

[4] D. Naso, F. Cupertino, B. Turchiano. Precise position control of tubular linear motors with neural networks and composite learning. Control Engineering Practice, vol. 18, no. 5, pp. 515-522, 2010.

[5] M. C. Zhang, W. S. Yin, Y. Zhu. Force ripple modeling and suppression in permanent magnet linear synchronous motors. Journal of Tsinghua University (Science \& Technology), vol. 50, no. 8, pp.1253-1257, 2010.

[6] K. K. Tan, S. N. Huang, T. H. Lee. Robust adaptive numerical compensation for friction and force ripple in permanent magnet linear motors. IEEE Transactions on Magnetics, vol. 38, no. 1, pp. 221-228, 2002.

[7] B. S. Prasad, D. S. Prasad, A. Sandeep, G. Veeraiah. Condition monitoring of $\mathrm{CNC}$ machining using adaptive control. International Journal of Automation and Computing, vol. 10, no. 3, pp. 202-209, 2013.

[8] K. K. Tan, T. H. Lee, H. X. Zhou. Micro-positioning of linear-piezoelectric motors based on a learning nonlinear PID controller. IEEE/ASME Transactions on Mechatronics, vol. 6, no. 4, pp. 428-436, 2001.

[9] K. K. Tan. Precision motion control with disturbance observer for pulse width-modulated-driven permanent magnet linear motors. IEEE Transactions on Magnetics, vol. 39, no. 3, pp. 1813-1818, 2003.

[10] X. M. Zhao, Q. D. Guo. Robust 2DOF control for periodic reference signal and disturbance signal in piston machining. Transactions of China Electrotechnical Society, vol. 21, no. 3, pp. 123-126, 2006. (in Chinese)
[11] H. Li, M. Steurer, K. L. Shi, S. Woodruff, D. Zhang. Development of a unified design, test, and research platform for wind energy systems based on hardware-in-the-loop realtime simulation. IEEE Transactions on Industrial Electronics, vol. 53, no. 4, pp. 1144-1151, 2006.

[12] K. K. Tan, T. H. Lee, S. N. Huang, F. M. Leu. Adaptive predictive control of a class of SISO nonlinear systems. Dynamics and Control, vol. 11, no. 2, pp. 151-174, 2001.

[13] K. K. Tan, T. H. Lee, S. N. Huang, J. X. Xu. Friction modeling and adaptive compensation using a relay feedback approach. IEEE Transactions on Industrial Electronics, vol. 48, no. 1, pp. 169-176, 2001.

[14] Z. S. Hou, Z. Wang. From model-based control to datadriven control: Survey, classification and perspective. Information Sciences, vol. 235, pp. 3-35, 2013.

[15] Z. S. Hou. Nonparametric Models and Its Adaptive Control Theory, Beijing: Science Press, 1999.

[16] Z. S. Hou, S. T. Jin. Model Free Adaptive Control: Theory and Applications, Boca Raton: CRC Press, 2013.

[17] R. M. Cao, H. X. Zhou, Z. S. Hou. Data-driven model-free adaptive precision control for linear servo system. Control Theory and Applications, vol. 29, no. 3, pp.310-316, 2012. (in Chinese)

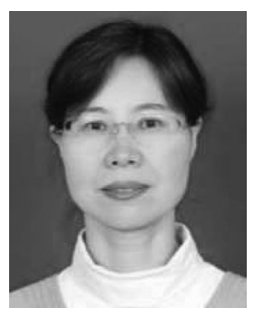

Rong-Min Cao graduated from Shaanxi University of Science \& Technology, China in 1986. She received the M. Sc. degree from Beijing Jiaotong University, China in 1996 and the Ph. D. degree from China Agricultural University in 2012. She is currently a professor at School of $\mathrm{Au}$ tomation of Beijing Information Science and Technology University.

Her research interests include datadriven control method and its application, and study and application of direct-drive technology.

E-mail: rongmin_cao@163.com (Corresponding author)

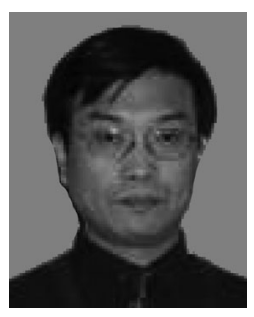

Zhong-Sheng Hou graduated from Jilin University of Technology, China in 1983. He received the M.Sc. degree from Jilin University of Technology in 1988, and Ph. D. degree from Northeast University, China in 1994. He received postdoctoral fellowship from Harbin University of Technology, China in 1997. He is now a professor at Beijing Jiaotong University.

His research interests include model free adaptive control, data-driven control, learning control, intelligent transportation system, and application of data mining in medical and traffic field.

E-mail: zhshhou@bistu.edu.cn

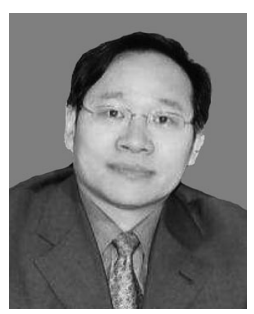

Hui-Xing Zhou graduated from Dalian University of Technology, China in 1983. $\mathrm{He}$ received the $\mathrm{Ph}$. D. degree from Tsinghua University, China 1998. He received postdoctoral fellowship from National University of Singapore, Singapore in 2001. He is a professor at China Agricultural University

His research interests include directdrive technology study and precision motion control, computer numerical control (CNC), the mechatronics product research and development.

E-mail: perc_zhx@126.com 